FTUV/94-72

IFIC/94-69

hep-ph 9502237

October 7, 2018

\title{
NOVEL SCALAR BOSON DECAYS IN SUSY WITH BROKEN R-PARITY
}

\author{
F. de Campos, 周 \\ M. A. García-Jareño, \\ Anjan S. Joshipura \\ J. Rosiek \\ and \\ José W. F. Valle \\ Instituto de Física Corpuscular - IFIC/C.S.I.C. \\ Dept. de Física Teòrica, Universitat de València \\ 46100 Burjassot, València, SPAIN
}

\begin{abstract}
$\mathrm{R}$ parity violation can induce mixing of the supersymmetric Higgs bosons with the sneutrinos at the tree level. We study the effect of this mixing on the decays of Higgs scalars as well as sneutrinos in an effective model where the violation of $R$ parity is included in the minimal supersymmetric model through bilinear lepton number violating superpotential terms. We show that a small violation of $R$ parity can lead to a sizeable branching ratio for the supersymmetric Higgs boson decay mode $H \rightarrow \chi \ell$ (where $\chi$ denotes an electroweak gaugino and $\ell$ is either a tau neutrino or a tau lepton). Relevant constraints on $R$ parity violation as well as those coming from SUSY particle searches still allow the decay $H \rightarrow \chi \ell$ to compete with the conventional decay $H \rightarrow b \bar{b}$, at least for some ranges of parameters of the model. Moreover, the tau sneutrino will have dominant $\mathrm{R}$ parity violating decays to standard model fermions $b \bar{b}, \tau^{+} \tau^{-}$or to the invisible mode $\nu \bar{\nu}$ whenever the phase space for $\mathrm{R}$ parity conserving channels is closed.
\end{abstract}

\footnotetext{
*E-mail CAMPOSC@evalvx.ific.uv.es

${ }^{\dagger}$ E-mail GARCIAMA@evalvx.ific.uv.es

${ }^{\ddagger}$ E-mail anjan@prl.ernet.in

§E-mail ROSIEK@evalvx.ific.uv.es and ROSIEK@fuw.edu.pl

`E-mail VALLE@flamenco.ific.uv.es and VALLE@evalvx.ific.uv.es
} 


\section{Introduction}

The minimal supersymmetric standard model (MSSM) [1] provides an attractive generalization of the standard electroweak theory and its phenomenology is quite well studied [2]. In analysing the consequences of the MSSM one usually assumes [3] the conservation of a discrete $Z_{2}$ symmetry, called $\mathrm{R}$ parity, distinguishing matter fields from their superpartners. Given the importance of this assumption and the fact that it does not follow on any general grounds, it is important to analyse the possible implications of relaxing it in phenomenological studies of supersymmetric models. Considerable effort has gone into studying consequences of $\mathrm{R}$ parity violating versions of the MSSM [4][10], as well its extensions which realize the spontaneous violation of $R$ parity [11, 12]. The breakdown of $\mathrm{R}$ parity and that of the $S U(2) \otimes U(1)$ symmetry can result in the mixing of the ordinary particles with the superparticles having the same electric charge and spin. Thus the $\mathrm{R}$ parity violation could lead to mixing of $(i)$ gauginos $(\lambda)$ with leptons $(\ell)$ and $(i i)$ Higgs bosons with sleptons $(\tilde{\ell})$. The consequences of the gaugino-lepton mixings have been analysed in many papers [5, 7]. Such mixing leads to neutrino masses $4,10,13$ and decays [14, as well as to $\mathrm{R}$ parity violating $\mathrm{Z}$ decays such as $Z \rightarrow \lambda+\ell[12]$.

In contrast to the $\lambda-\ell$ mixing, the consequences of the mixing of Higgs bosons with sleptons are relatively less explored [8, 9, 10. This mixing could have important implications in the Higgs sector. For example, it was shown recently in [9], that one could violate CP spontaneously in the MSSM as a consequence of such mixing. Here we study an important consequence of the $H-\tilde{\nu}$ mixing, namely, the possibility of Higgs boson decays into gauginos and leptons. These decays differ from the corresponding $\mathrm{R}$ parity violating $Z$ decays in an important way.

Various experimental constraints on the magnitude of $\mathrm{R}$ parity violation restrict the $\chi-\ell$ mixing and hence the attainable values of the branching ratios for $\mathrm{R}$ parity violating $\mathrm{Z}$ decays to be at the level of $\mathcal{O}\left(10^{-5}\right)$ or so [12] and therefore within the sensitivities of the LEP experiments. Similar constraints also limit the amount of mixing between the Higgs bosons and the sleptons. However, in contrast to the Higgs boson, which mainly decays through Yukawa couplings, the sleptons decay through gauge interactions. This can compensate for the smallness of the Higgs-slepton mixing, opening the possibility for sizeable $\mathrm{R}$ parity violating Higgs boson decays. 
In fact, as we will show explicitly, if allowed kinematically, the SUSY decay e.g. $H \rightarrow \chi \ell$ ( $\chi$ being here lightest neutralino, often assumed to be also the lightest supersymmetric particle - or LSP, and $\ell$ being in this case the $\nu_{\tau}$ ) can become comparable or even dominate over the conventional decay to $b \bar{b}$ pair for some ranges of SUSY parameters.

The $H-\tilde{\nu}$ mixing can also have sizeable effects on the sneutrino decay pattern as well. If, as usually assumed in the MSSM, one of the neutralinos is the lightest supersymmetric particle, the sneutrinos will decay to the LSP and a neutrino. If not, then the sneutrinos will only have three body decay modes. In contrast, in the present case, if all neutralinos are heavier than the sneutrino, the latter would dominantly decay to non supersymmetric channels, for example $b \bar{b}$ pairs (through the $H-\tilde{\nu}$ mixing) or to $\nu \bar{\nu}$ pairs (through $\chi^{0}-\nu$ mixing). Similar decays to tau pairs would also be induced by $\chi-\tau$ mixing. The existence of such new sneutrino decay modes can significantly influence the conventional strategy for sneutrino search.

In this note, we explore the interesting range of the allowed parameter space of the MSSM for which the decay $H \rightarrow \chi \ell$ is comparable to the standard decay mode $H \rightarrow b \bar{b}$ and discuss the possible signatures associated with this SUSY decay mode. We also show that, quite generally, the $R$ parity violating sneutrino decay channels to standard model fermions are dominant below the threshold for $\mathrm{R}$ parity conserving supersymmetric decay channels.

\section{Basic Framework}

The MSSM is characterised by the following superpotential, written in standard notation:

$$
W_{0}=\varepsilon_{a b}\left[h_{i j} \hat{L}_{i}^{a} \hat{H}_{1}^{b} \hat{E}_{j}^{C}+h_{i j}^{\prime} \hat{Q}_{i}^{a} \hat{H}_{1}^{b} \hat{D}_{j}^{C}+h_{i j}^{\prime \prime} \hat{Q}_{i}^{a} \hat{H}_{2}^{b} \hat{U}_{j}^{C}+\mu \hat{H}_{1}^{a} \hat{H}_{2}^{b}\right]
$$

One could also add to this the following $\mathrm{R}$ violating terms

$$
W_{R}=\varepsilon_{a b}\left[\lambda_{i j k} \hat{L}_{i}^{a} \hat{L}_{j}^{b} \hat{E}_{k}^{C}+\lambda_{i j k}^{\prime} \hat{L}_{i}^{a} \hat{Q}_{j}^{b} \hat{D}_{k}^{C}+\epsilon_{i} \hat{L}_{i}^{a} \hat{H}_{2}^{b}\right]
$$

where we have omitted the baryon number violating terms [3] whose presence along with terms in eq. (2) would lead to fast proton decay. 
In what follows we will focus only on the effect of the bilinear $\mathrm{R}$ parity violating term in eq. (2), which is the only one to have a direct effect on the physics of the neutral Higgs boson sector. This term, characterised by strength parameters $\epsilon_{i}$, plays a crucial role in generating Higgs-slepton and gaugino-lepton mixing at the tree level.

The scalar potential contains the standard soft supersymmetry breaking as well as supersymmetric terms following from the superpotential in eq. (1) and eq. (2). The neutral part of the potential can be written as

$$
\begin{aligned}
V_{\text {Higgs }} & =m_{1}^{2}\left|\phi_{1}\right|^{2}+m_{2}^{2}\left|\phi_{2}\right|^{2}+m_{L_{i}}^{2}\left(\tilde{\nu}_{i}^{\dagger} \tilde{\nu}_{i}\right)+\lambda\left(\left|\phi_{1}\right|^{2}-\left|\phi_{2}\right|^{2}+\tilde{\nu}_{i}^{\dagger} \tilde{\nu}_{i}\right)^{2} \\
& +\left[\mu \epsilon_{i} \phi_{1} \tilde{\nu}_{i}^{*}+B_{1} \mu m_{3 / 2} \phi_{1} \phi_{2}+B_{2} \epsilon_{i} m_{3 / 2} \phi_{2} \tilde{\nu}_{i}+\text { h.c. }\right]
\end{aligned}
$$

where $\phi_{1,2} \equiv H_{1,2}^{0}$ and $\lambda=\frac{1}{8}\left(g^{2}+g^{\prime 2}\right)$, with $g$ and $g^{\prime}$ denoting the $S U(2)$ and $U(1)$ couplings. The terms involving parameters $\epsilon_{i}$ are generated by the $\mathrm{R}$ violating terms in eq.(2). As a result of these terms, sneutrino fields invariably acquire a nonzero VEV «, 8, 15]. For definiteness we assume that only $\epsilon_{3}$ is non-zero".

Above the electroweak (and supersymmetry) breaking scale it is always possible to redefine the $\hat{L}_{3}$ and $\hat{H}_{1}$ superfields so as to eliminate the bilinear term $\hat{L}_{3} \hat{H}_{2}$ from the superpotential. However this does not mean that an analogous term is not present in the scalar potential. Indeed, the redefinition of $\hat{L}_{3}$ and $\hat{H}_{1}$ through the orthogonal transformation

$$
\begin{array}{r}
\hat{H}_{1}^{\prime}=\frac{\mu \hat{H}_{1}+\varepsilon_{3} \hat{L}_{3}}{\sqrt{\mu^{2}+\varepsilon_{3}^{2}}} \\
\hat{L}_{3}^{\prime}=\frac{-\varepsilon_{3} \hat{H}_{1}+\mu \hat{L}_{3}}{\sqrt{\mu^{2}+\varepsilon_{3}^{2}}}
\end{array}
$$

does not leave the scalar supersymmetry breaking mass terms invariant, since they are not expected to be universal below the unification scale. This implies that, after the substitution made in order to remove the $\hat{L}_{3} \hat{H}_{2}$ term from superpotential, one generates a linear term in the scalar potential for the slepton field, as a result of which a non-zero VEV for the scalar neutrino is induced.

The potential in eq. (3) is minimized for

$$
v_{1}\left[m_{1}^{2}+\lambda c\right]+B_{1} \mu m_{3 / 2} v_{2}+\mu \epsilon_{3} v_{3}=0
$$

\footnotetext{
$\|$ By neglecting $\epsilon_{1}$ and $\epsilon_{2}$ we are safe from the point of view of possible baryogenesis constraints [16]
} 


$$
\begin{aligned}
v_{2}\left[m_{2}^{2}-\lambda c\right]+B_{1} \mu m_{3 / 2} v_{1}+B_{2} \epsilon_{3} m_{3 / 2} v_{3} & =0 \\
v_{3}\left[m_{L_{3}}^{2}+\lambda c\right]+\epsilon_{3}\left[B_{2} m_{3 / 2} v_{2}+\mu v_{1}\right] & =0
\end{aligned}
$$

where $\left\langle\phi_{1}\right\rangle \equiv \frac{v_{1}}{\sqrt{2}},\left\langle\phi_{2}\right\rangle \equiv \frac{v_{2}}{\sqrt{2}},\left\langle\tilde{\nu_{3}}\right\rangle \equiv \frac{v_{3}}{\sqrt{2}}$ and $c \equiv\left(v_{1}^{2}-v_{2}^{2}+v_{3}^{2}\right)$. The presence of a nonzero $v_{3}$ leads to mixing of the scalar (pseudoscalar) Higgs with the scalar (pseudoscalar) component of the sneutrino. The relevant mass matrices are now given by

$$
M_{R}^{2}=\left(\begin{array}{ccc}
m_{1}^{2}+\lambda c+2 \lambda v_{1}^{2} & -\lambda v_{1} v_{2}+B_{1} \mu m_{3 / 2} & \lambda v_{1} v_{3}+\mu \epsilon_{3} \\
-\lambda v_{1} v_{2}+B_{1} \mu m_{3 / 2} & m_{2}^{2}-\lambda c+2 \lambda v_{2}^{2} & -\lambda v_{3} v_{2}+B_{2} \epsilon_{3} m_{3 / 2} \\
\lambda v_{1} v_{3}+\mu \epsilon_{3} & -\lambda v_{3} v_{2}+B_{2} \epsilon_{3} m_{3 / 2} & m_{L_{3}}^{2}+\lambda c+2 \lambda v_{3}^{2}
\end{array}\right)
$$

The $\epsilon_{3}$-dependent terms in the above equation lead to Higgs-sneutrino mixing and causes the $\mathrm{R}$ parity violating Higgs bosons decay modes. In order to see the effects of these decay modes note that the decay of Higgs to $\chi$ and $\nu$ occurs either through the sneutrino component of the lightest Higgs boson $\equiv h$,

$$
h=a_{31}(\tilde{\nu})_{R}+a_{21}\left(\phi_{2}\right)_{R}+a_{11}\left(\phi_{1}\right)_{R}
$$

(where the subscript $R$ denotes the real part), or through the $\nu_{\tau}$ admixture in the lightest neutralino in the $h \chi \chi$ vertex.

The relative importance of the SUSY decay mode $h \rightarrow \chi \nu$ follows from the ratio

$$
R_{0} \approx \frac{\Gamma(h \rightarrow \chi \nu)}{\Gamma(h \rightarrow b \bar{b})}=\frac{\tan ^{2} \theta_{W}}{2}\left(\frac{M_{W}}{m_{b}}\right)^{2}\left(\frac{\left(1-m_{\chi}^{2} / M_{H}^{2}\right)^{2}}{\left(1-4 m_{b}^{2} / M_{H}^{2}\right)^{3 / 2}}\right) \frac{a_{31}^{2}}{a_{11}^{2}} \cos ^{2} \beta|\xi|^{2}
$$

where $\xi$ denotes the appropriate gaugino mixing factor specifying the direction of the LSP. Although less likely kinematically, one may also have decays of supersymmetric Higgs bosons into charginos and taus. The relative importance of this decay mode follows from the ratio

$$
R_{+} \approx \frac{\Gamma\left(h \rightarrow \chi^{+} \tau^{-}+\chi^{-} \tau^{+}\right)}{\Gamma(h \rightarrow b \bar{b})}=\frac{2}{3}\left(\frac{M_{W}}{m_{b}}\right)^{2}\left(\frac{\left(1-m_{\chi}^{2} / M_{H}^{2}\right)^{2}}{\left(1-4 m_{b}^{2} / M_{H}^{2}\right)^{3 / 2}}\right) \frac{a_{31}^{2}}{a_{11}^{2}} \cos ^{2} \beta\left|\xi^{\prime}\right|^{2}(10)
$$


where $\xi^{\prime}$ is the wino-lightest chargino mixing element.

Similarly one can induce tau sneutrino decays to normal standard model fermions due to $\mathrm{R}$ parity breaking either in the scalar sector or in the chargino or neutralino sectors. As we will discuss later, the tau sneutrino will have dominant $\mathrm{R}$ parity violating decays to standard model fermions whenever the phase space for $\mathrm{R}$ parity conserving channels is closed.

In determining these branching ratios one has also to identify mass eigenstate charged and neutral fermions, which follow from the corresponding neutralino and chargino mass matrices. First we give the neutralino matrix, expressed in the basis $-i \tilde{B},-i \tilde{W}_{3}, \tilde{h_{1}^{0}}, \tilde{h_{2}^{0}}, \nu_{\tau}$ :

$$
M_{\chi^{0}}=\left(\begin{array}{ccccc}
x M & 0 & -\frac{g^{\prime} v_{1}}{2} & \frac{g^{\prime} v_{2}}{2} & -\frac{g^{\prime} v_{3}}{2} \\
0 & M & \frac{g v_{1}}{2} & -\frac{g v_{2}}{2} & \frac{g v_{3}}{2} \\
-\frac{g^{\prime} v_{1}}{2} & \frac{g v_{1}}{2} & 0 & -\mu & 0 \\
\frac{g^{\prime} v_{2}}{2} & -\frac{g v_{2}}{2} & -\mu & 0 & \epsilon_{3} \\
-\frac{g^{\prime} v_{3}}{2} & \frac{g v_{3}}{2} & 0 & \epsilon_{3} & 0
\end{array}\right)
$$

where $M$ denotes the gaugino mass and we assumed the GUT unification hypothesis $x=\frac{5 g^{\prime 2}}{3 g^{2}}=\frac{5}{3} \tan ^{2} \theta_{W} \sim 0.5$

For charginos one has the following mass matrix $M_{\chi^{ \pm}}$, written in the basis where the rows correspond to $\left(-i \tilde{W}^{-}, \tilde{h_{1}^{-}}, \tau_{L}^{-}\right)$while the columns stand for $\left(-i \tilde{W}^{+}, \tilde{h_{2}^{+}}, \tau_{R}^{+}\right)$:

$$
M_{\chi^{ \pm}}=\left(\begin{array}{ccc}
M & \frac{g v_{1}}{\sqrt{2}} & \frac{g v_{3}}{\sqrt{2}} \\
\frac{g v_{2}}{\sqrt{2}} & \mu & -\epsilon_{3} \\
0 & \frac{h_{33} v_{3}}{\sqrt{2}} & \frac{-h_{33} v_{1}}{\sqrt{2}}
\end{array}\right)
$$

From the above formulas one expects that a modest mixing $(\sim 20 \%)$ between the Higgs boson and the sneutrino can give rise to a sizeable branching ratio for $\mathrm{R}$ parity violating Higgs bosons decay modes. The same phenomena may also occur in the decays of the pseudoscalar Higgs bosons. This will happen for moderate $\epsilon_{3}$ values (which correspond to phenomenologically acceptable $\nu_{\tau}$ masses), if the sneutrino mass is close to the mass 
of the Higgs boson, where by sneutrino we mean a state that is more than $50 \%$ along the original weak basis sneutrino, with the corresponding labeling for the Higgs case. As we will demonstrate, it is indeed possible to obtain the required amount of mixing satisfying all the relevant constraints on the magnitude of the $R$ parity breaking that follow from experiment.

\section{Constraints}

We now turn to a more detailed analysis which includes all relevant constraints on the parameters. The major constraint on $\epsilon_{3}$ and hence on $\mathrm{R}$ parity violating mixings comes from the neutrino mass. Both the non-zero $\epsilon_{3}$ and $v_{3}$ induce the mass for the $\nu_{\tau}$. This follows from the structure of the neutralino mass matrix $M_{\chi}$ in eq. (11). This matrix is of the seesaw form and results in the following mass for the neutrinos [10]

$$
m_{\nu_{\tau}} \approx \frac{x g^{2}+g^{\prime 2}}{2 \mu} \frac{\left(\mu v_{3}+v_{1} \epsilon_{3}\right)^{2}}{\left[-2 x \mu M-v_{1} v_{2}\left(x g^{2}+g^{\prime 2}\right)\right]}
$$

Since $m_{\nu_{\tau}}$ is constrained to be $\leq 31 \mathrm{MeV}$ [17], $\epsilon_{3}$ is correspondingly restricted. The constraints on $R$ parity conserving parameters come from the non-observation of the SUSY particles at LEP1.

Using the minimization equation, one could express all the elements of the various mixing matrices in terms of six independent parameters which we choose as $\tan \beta=\frac{v_{2}}{\sqrt{v_{1}^{2}+v_{3}^{2}}}$, the $\mu$ parameter, the pseudoscalar mass parameter $m_{A}^{2} \equiv m_{1}^{2}+m_{2}^{2}$, the gaugino mass parameter $M$, the soft sneutrino mass parameter $m_{L_{3}}$ 网, and, finally, the parameter $\epsilon_{3}$. This last parameter $\epsilon_{3}$ is absent in the standard $R$ parity conserving MSSM case. As we will see, this parameter is directly constrained by the tau neutrino mass.

In our present analysis we have taken into account the following constraints on the model parameters:

- The tau neutrino mass bound following from the direct searches in the laboratory [17]. Requiring $m_{\nu_{\tau}}$ to be $\leq 31 \mathrm{MeV}$ restricts the parameters in eq. (13). On the other hand, the cosmological arguments based on the closure density impose much

\footnotetext{
**For simplicity we choose the parameter $\gamma \equiv \frac{B_{2}}{B_{1}}$ to be 1
} 
stronger constraints [18]. Indeed, a stable tau neutrino heavier than about 50 $\mathrm{eV}$ is cosmologically forbidden. However, this problem is easily solved in models where the violation of $\mathrm{R}$ parity is spontaneous, since in this case the theory contains a Goldstone boson, called majoron [19, 20]. The $\nu_{\tau}$ is then unstable, decaying to a lighter neutrino plus majoron [21, 22] and thereby avoiding an excessive relic neutrino abundance [14]. Another cosmological constraint follows from big bang nucleosynthesis [23]. However it seems reasonable to consider the less conservative limit of $31 \mathrm{MeV}$ on $m_{\nu_{\tau}}$, since, even in the absence of a majoron, the off-diagonal coupling of the $Z$ to $\nu_{\tau}$ and a lighter neutrino can lead to a cosmologically safe decay for the $\nu_{\tau}$ into three neutrinos [21, 24]. Note that such couplings would arise in the present case once the parameters $\epsilon_{1,2}$ (assumed here to be zero for simplicity) are turned on. Although much less efficient than the majoron decay channel, this decay is enough for the case of large $\nu_{\tau}$ masses, where the $\mathrm{R}$ parity violating effects discussed here can be sizeable.

- The lightest of the charginos should be heavier than $\sim 45 \mathrm{GeV}$ as required by present negative result of supersymmetric searches at LEP.

- Z decays to chargino and neutralinos may affect the partial Z decay widths, which should obey the present restrictions imposed by LEP. For the case of charginos this constraint follows automatically from to the previous one, while for the neutralino case it depends on SUSY parameter values

We have scanned the region of parameter space which is consistent with the above constraints and for which the decay of supersymmetric Higgs bosons to LSP and neutrino $\left(H \rightarrow \chi^{0}+\nu_{\tau}\right)$ is kinematically allowed. For definiteness, we have restricted our considerations to the case of $\mathrm{CP}$ even Higgs bosons. The $\mathrm{R}$ parity violating decays of pseudoscalar Higgs bosons $A \rightarrow \chi^{0}+\nu_{\tau}$ and $A \rightarrow \chi^{ \pm}+\tau^{\mp}$ can be investigated in a similar way.

The mixing $a_{31}$ appearing in eq. (9) and the analogous one for the pseudoscalar case will become significant if the sneutrino mass is close to the mass of the relevant Higgs boson. 


\section{Results}

Our results for R parity violating supersymmetric Higgs boson decays are summarized explicitly in figures 1 to 6 .

In fig. 1 we display the branching ratios for CP even Higgs decays to LSP plus neutrino as a function of the relevant Higgs boson-sneutrino mass difference, for a suitable choice of SUSY parameters, specified in the figure. In fig. 2 we display the corresponding "standard" $b \bar{b}$ decay branching ratio for the same illustrative choice of parameters. Clearly, for relatively small Higgs boson-sneutrino mass differences of a few $\mathrm{GeV}$ or so, the supersymmetric channel can dominate over the standard one.

Conversely, it is possible in our model to substantially affect sneutrino decay patterns, as the tau sneutrino may decay into $\mathrm{R}$ parity violating standard model channels such as $b \bar{b}, \tau^{+} \tau^{-}$or the invisible mode $\nu \bar{\nu}$. Indeed, these decays are the dominant ones whenever the phase space for the $\mathrm{R}$ parity conserving channels such as $\chi \nu$ is closed. This is seen from figures 3, 4 and 5. For example, for the choice of parameters given in fig. 3 the mass of the lightest chargino is around $120 \mathrm{GeV}$. This explains the drop in the branching ratio for sneutrino to $\nu \bar{\nu}$ at this value of the sneutrino mass as, above this threshold, the supersymmetric decay channel would be open. This illustrates how the sneutrino decay branching ratios into non-supersymmetric channels can be sizeable when the supersymmetric channels are kinematically forbidden. On the other hand, fig. 4 illustrates that there is a resonant enhancement of the $b \bar{b}$ and $\tau^{+} \tau^{-}$decay branching ratios for sneutrino masses close to the lightest CP even Higgs boson mass.

The same effects can also be seen from fig. 5 , which corresponds to the $\tan \beta=10$, $M_{2}=70 \mathrm{GeV}, \mu=200 \mathrm{GeV}, \epsilon_{3}=1$ and $m_{A}=250 \mathrm{GeV}$. Clearly, for sneutrino masses below the threshold for LSP production (about $60 \mathrm{GeV}$ in this case) the $\mathrm{R}$ parity violating standard model channels such as $b \bar{b}$ are dominant. Moreover, they may be nonnegligible even above the supersymmetric threshold, provided the sneutrino masses lies close to the lightest CP even Higgs boson mass, leading to a resonant enhancement of the $b \bar{b}$ or $\tau^{+} \tau^{-}$decay branching ratios as discussed above. For the parameter set used in fig. 5 this corresponds to $m_{h}=155 \mathrm{GeV}$ and $m_{\tilde{\nu}_{\tau}} \approx 150 \mathrm{GeV}$ and leads to the small rise which can be seen from this figure. Note also that, for tau sneutrino masses below the supersymmetric threshold, although the sneutrino will be the LSP, it clearly 
will be unstable, since $\mathrm{R}$ parity is broken, leading to visible signals (such as a $b \bar{b}$ pair), not necessarily missing momentum. These novel features may be quite important in designing strategies to search for the sneutrinos in our model.

As a final remark to close this section we note that there is a wide class of processes which exhibit R parity violation outside Higgs boson sector [25]. For definiteness, we calculate in this model, as an example, the corresponding $\mathrm{R}$ parity violating $\mathrm{Z}$ decay branching ratios [12]. We focus on the most characteristic one, namely single chargino production in $\mathrm{Z}$ decays. In fig. 6 we display the corresponding branching ratios for $\mathrm{Z}$ decays to the lightest chargino plus a tau of either charge, as a function of the relevant $\nu_{\tau}$ mass, after varying the SUSY parameters over a reasonable range, specified as $-250 \mathrm{GeV}<\mu<250 \mathrm{GeV}$ and $30 \mathrm{GeV}<M_{2}<200 \mathrm{GeV}$ and fixing the remaining relevant parameters as follows: $\tan \beta=5, m_{A}=50 \mathrm{GeV}, m_{\tilde{\nu}}=200 \mathrm{GeV}$, $\gamma=1$. Clearly, in this model these branching ratios are within the reach of the LEP experiments, for the same range of $\epsilon_{3}$ or $m_{\nu_{\tau}}$ masses for which the novel scalar boson decays modes suggested here are also sizeable.

\section{Discussion}

We have demonstrated in this paper that for some ranges of parameters of the MSSM model, the supersymmetric decay modes of the Higgs bosons can be comparable to their conventional decays. This feature can influence the supersymmetric Higgs boson search strategies in a substantial way. Note that the $\mathrm{R}$ parity breaking terms responsible for Higgs-sneutrino mixing also lead to the decay of the LSP $\chi$. Therefore the neutralino emitted in Higgs decays would be unstable and decay inside the detector if $\epsilon_{3} \sim$ few $\mathrm{GeV}$. The LSP decay modes in this case will consist of lepton + fermion-anti-fermion pairs [12]. For example, the R parity violating Higgs boson decays to the LSP, followed by the charged or neutral current mediated LSP decays would give rise to (a) two jets + missing momentum, (b) di-lepton + missing momentum, or (c) $\tau-e$ or $\tau-\mu$ pairs accompanied by missing momentum signatures. Folding now with the standard decays of the virtual $Z^{0}$ one gets the following signatures in $e^{+} e^{-}$collisions: (i) two jets + missing momentum, (ii) di-lepton + missing momentum, (iii) di-lepton plus di-jets + missing momentum, (iv) four jets + missing momentum, (v) four leptons 
+ missing momentum, (vi) $\tau^{+} \tau^{-}$pairs plus two jets, and (vii) $\tau-e$ or $\tau-\mu$ pairs accompanied by missing momentum. Some of these signals, for example (vii), do not occur in the standard model and, therefore, may be regarded as characteristic of our supersymmetric decay modes. It also does not appear in the MSSM, thus it appears to be a characteristic feature of the broken $\mathrm{R}$ parity model. For a sizeable branching ratio of $H \rightarrow \chi \nu$, these signals may be sizeable. Moreover, there may be, for some choice of parameters, the possibility of Higgs boson decays to charginos, which may also lead to novel signatures.

We have performed our study in the framework of the simplest model with $\mathrm{R}$ parity violation introduced in an explicit way, aware of the fact that this may face some stringent restrictions from nucleosynthesis, which would be interesting to evaluate in detail, taking into account the neutral current mediated decay of the $\nu_{\tau}$ into three neutrinos. We feel justified in using this as a reasonable working model, since the novel decays discussed here are also expected in a very general class of models with spontaneous violation of $\mathrm{R}$ parity, in which the cosmological restrictions are easily evaded due to the majoron decays or annihilations of the tau neutrino [14].

Our study opens the issues of how the existence of these novel scalar decay channels affect the bounds on the Higgs masses from the LEP experiments and of how they can influence the search strategies for Higgs bosons or sneutrinos at the higher energies which will be available at LEP200 or LHC. We plan to return to these issues elsewhere.

Finally, we re-stress that striking $\mathrm{R}$ parity violating effects are also expected to occur in the electroweak gauge currents, leading to sizeable $\mathrm{R}$ parity violating $\mathrm{Z}$ decays, as illustrated in fig. 6. Similary, $\mathrm{R}$ parity violating processes could be visible at hadron colliders such as the upcoming generation of Fermilab experiments and the LHC.

\section{Acknowledgements}

This work has been supported by DGICYT under Grant numbers PB92-0084 and SAB94-0014 (A.S.J.) as well as a postdoctoral fellowship (J.R.) and an FPI fellowship (M.A.G.J.). The work of F. de Campos was supported by CNPq (Brazil). 


\section{Figure Captions}

\section{Figure 1}

Branching ratio of the lightest Higgs boson into $\chi \nu_{\tau}$ as a function of the difference of the lightest Higgs boson and the sneutrino mass. We have fixed the supersymmetric parameters $\mu, \tan \beta, M_{2}$ and the pseudoscalar mass $m_{A}$ as shown in the figure. The $\nu_{\tau}$ mass was varied as indicated by the different curves in the figure.

\section{Figure 2}

Branching ratio of the lightest Higgs boson into $b \bar{b}$ as as a function of the difference of the lightest Higgs boson and the sneutrino mass. The values of the parameters as well as the $\nu_{\tau}$ mass are exactly as in the previous figure.

\section{Figure 3}

Branching ratio of the sneutrino into $\nu \bar{\nu}$ as a function of the sneutrino mass for five different values for the tau neutrino mass with the supersymmetric parameters fixed as indicated in the figure.

\section{Figure 4}

Branching ratio of the sneutrino into $b \bar{b}$ as a function of the difference of the lightest Higgs boson and the sneutrino mass. Parameters are chosen as in fig. 3 .

\section{Figure 5}

Branching ratio for sneutrino decays to $\mathrm{R}$ parity breaking channels and supersymmetric channels, as a function of the sneutrino mass. Parameters are chosen as indicated in the text.

\section{Figure 6}

$Z \rightarrow \chi \tau$ branching ratio for different values of the lightest chargino and $\nu_{\tau}$ masses, with the supersymmetric parameters chosen as described in the text. The area under each line is the allowed region giving a branching ratio in excess of the indicated value. All points satisfy the observational constraints that follow from SUSY particle searches as well as neutrino physics (see text). 


\section{References}

[1] H. P. Nilles, Phys. Rep. 110 (1984) 1; for recent review see X. Tata in The Standard Model and Beyond p. 304, ed. J. E. Kim, World Scientific 1991.

[2] See for example, H. E. Haber and G. L. Kane, Phys. Rep. 117 (1985) 75; ALEPH collaboration, Phys. Rep. 216 (1992) 253; S. Dawson, Nucl. Phys. B261 (1985) 297.

[3] S. Weinberg, Phys. Rev. D26 (1982) 533; N. Sakai and T. Yanagida, Nucl. Phys. B197 (1982) 533;

[4] L. Hall and M. Suzuki, Nucl. Phys. B231 (1984) 419; I. Lee Nucl. Phys. B246 (1984) 120

[5] G. G. Ross and J. W. F. Valle, Phys. Lett. B151 (1985) 375; J. Ellis et al., Phys. Lett. B150 (1985) 142.

[6] R. M. Godbole, P. Roy and X. Tata, Nucl. Phys. B401 (1993) 67

[7] For a recent review see D. P. Roy, invited talk at the X Symposium in High Energy Physics, Bombay, TIFR preprint TIFR/TH/93-14.

[8] D. Comelli, A. Masiero, M. Pietroni and A. Riotto, Phys. Lett. B224 (1994) 397

[9] A. S. Joshipura and M. Nowakowski, PRL-TH-94/11, hep-ph 9403349

[10] A. S. Joshipura and M. Nowakowski, FTUV/94-42, IFIC/94-37, hep-ph 9408224 (Phys. Rev. D, to appear)

[11] A Masiero, J. W. F. Valle, Phys. Lett. B251 (1990) 273; J. C. Romao, C. A. Santos, and J. W. F. Valle, Phys. Lett. B288 (1992) 311; G. Giudice, A. Masiero, M. Pietroni, A. Riotto, Nucl. Phys. B396 (1993) 243; M. Shiraishi, I. Umemura, K. Yamamoto, Phys. Lett. B313 (1993) 89

[12] P. Nogueira, J. C. Romão, J. W. F. Valle, Phys. Lett. B251 (1990) 142; R. Barbieri, L. Hall, Phys. Lett. B238 (1990) 86; M. C. Gonzalez-Garcia, J. W. F. Valle, Nucl. Phys. B355 (1991) 330; D. E. Brahm et al., Phys. Rev. D42 (1992) 1860 
[13] K. Enqvist, A. Masiero and A. Riotto, Nucl. Phys. B373 (1992) 95; E. Roulet and D. Tommasini, Phys. Lett. B256 (1991) 218; D. Tommasini, Phys. Lett. B297 (1992) 125.

[14] J. C. Romão and J. W. F. Valle Phys. Lett. B272 (1991) 436; Nucl. Phys. B381 (1992) 87.

[15] C Aulakh, R Mohapatra, Phys. Lett. B119 (1983) 136; A Santamaria, J W F Valle, Phys. Lett. B195 (1987) 423; Phys. Rev. Lett. 60 (1988) 397; Phys. Rev. D39 (1989) 1780

[16] H. Dreiner and G. G. Ross Nucl. Phys. B410 (1993) 188 and references therein

[17] Particle Data Group, Phys. Rev. D50 (1994) 1173

[18] E. Kolb, M. Turner, The Early Universe, Addison-Wesley, 1990.

[19] Y. Chikashige, R. Mohapatra, R. Peccei, Phys. Rev. Lett. 45 (1980) 1926

[20] For a recent review see J. W. F. Valle, Gauge Theories and the Physics of Neutrino Mass, Prog. Part. Nucl. Phys. 26 (1991) 91-171 (ed. A. Faessler), and references therein.

[21] J. Schechter and J. W. F. Valle, Phys. Rev. D25 (1982) 774;

[22] J. W. F. Valle, Phys. Lett. B131 (1983) 87; G. Gelmini, J. W. F. Valle, Phys. Lett. B142 (1984) 181; M. C. Gonzalez-Garcia, J. W. F. Valle, Phys. Lett. B216 (1989) 360. A. Joshipura, S. Rindani, PRL-TH/92-10

[23] For a review see G. Steigman; proceedings of the International School on Cosmological Dark Matter, (World Scientific, 1994), ed. J. W. F. Valle and A. Perez, p. 55

[24] J. Schechter and J. W. F. Valle, Phys. Rev. D22 (1980) 2227

[25] J. W. F. Valle, Physics at New Accelerators, Looking Beyond the Standard Model, invited talk at Neutrino 92 Nucl. Phys. B (Proc. Suppl.) 31 (1993) 221-232 
This figure "fig1-1.png" is available in "png" format from: http://arxiv.org/ps/hep-ph/9502237v2 
This figure "fig1-2.png" is available in "png" format from: http://arxiv.org/ps/hep-ph/9502237v2 
This figure "fig1-3.png" is available in "png" format from: http://arxiv.org/ps/hep-ph/9502237v2 
This figure "fig1-4.png" is available in "png" format from: http://arxiv.org/ps/hep-ph/9502237v2 
This figure "fig1-5.png" is available in "png" format from: http://arxiv.org/ps/hep-ph/9502237v2 